\title{
A Cost-efficient Model for Predicting Cerebral A $\beta$ Burden Using MRI and Neuropsychological Markers in the ADNI-2 Cohort: Comparisons in Predictability among Machine Learning Models
}

\author{
Hyunwoong Ko ${ }^{1,3, *}$, Seho Park ${ }^{2}$, Jungjoon Ihm ${ }^{1,3,4,}$, and for the ADNI Research Group \\ ${ }^{1}$ Interdisciplinary Program in Cognitive Science, Seoul National University, Seoul, South Korea \\ ${ }^{2}$ School of Medicine, Boston University, Boston, MA, United States \\ ${ }^{3}$ Dental Research Institute, Seoul National University, School of Dentistry, Seoul, Korea \\ ${ }^{4}$ School of Dentistry, Seoul National University, Seoul, South Korea \\ * Correspondence: powerzines@snu.ac.kr (H.K.); ijj127@snu.ac.kr (J.I.)
}

\begin{abstract}
Substantial studies have focused on early detection of Alzheimer's disease (AD). Cerebral amyloid beta $(\mathrm{A} \beta)$, is hallmark of $\mathrm{AD}$, can be observed in vivo via positron emission tomography imaging using amyloid tracer or cerebrospinal fluid assessment, but costly expensive. The current study aims to identify and compared predictability in magnetic resonance imaging (MRI) markers and neuropsychological markers to predict cerebral $\mathrm{A} \beta$ status in $\mathrm{AD}$ cohort using machine learning (ML) approaches. The predictability in candidate markers for cerebral $\mathrm{A} \beta$ status was examined by analyzing 724 participants from the ADNI-2 cohort. Demographic variables, structural MRI markers, and neuropsychological test scores were used as input in several ML algorithms to predict cerebral A $\beta$ positivity. Out of five combination of candidate markers, neuropsychological markers with demographics showed the most cost-efficient result. A feature selection model could distinguish abnormal levels of $A \beta$ with the predictability of 0.85 , indicating the same performance with MRI-based models. The result has first to identified the predictability in MRI markers using ML approaches, and secondary to demonstrate the neuropsychological model with demographics could predict $A \beta$ positivity, suggesting a more cost-efficient method for detecting cerebral $A \beta$ status compared to MRI markers.
\end{abstract}

Keywords: amyloid beta, neuropsychological assessment, machine learning, Alzheimer's disease

\section{Introduction}

In 2010, 35.6 million people across the world were estimated to have dementia, which will approximately double to 65.7 million in 2030 [1]. With growing numbers of those with dementia, the cost of dementia can also be problematic. In the US the annual payment for dementia care was $\$ 236$ billion in 2016, being expected to increase to more than $\$ 1$ trillion in 2050 [2]. Alzheimer's disease (AD) is the most common cause of dementia, explaining about $70 \%$ of dementia population [3]. Consistently, the structural magnetic resonance imaging (MRI) has been used most frequently as a diagnostic modality for AD. It can help for clinician to allow for finding structurally pathological changes in the brain and the irreversible atrophy due to the neurodegeneration according to $\mathrm{AD}$ pathology [4]. A decade ago, the perspective about the disease was that either people with dementia had AD-related pathological changes, or those who were cognitively normal $(\mathrm{CN})$ did not have such changes in their brains [5]. A view of the disease, however, has been reconsidered, in which both AD related pathological changes and clinical symptoms occur constantly, suggesting that the end state of that process indicate dementia, with accumulation of pathological and clinical changes. In this respect, the structural MRI (e.g., cortical atrophy) as clinical information on AD had no longer specific 
evidence for $\mathrm{AD}$, but the extent of cortical atrophy measured by structural MRI still associates well with Braak staging at postmortem autopsy evidences [6, 7].

Recently, it has been well established that beta-amyloid $(\mathrm{A} \beta)$ is essential component in AD and $\mathrm{A} \beta$ begins to accrue almost 10-15 years before the clinical onset of AD [8]. Advance in AD research and imaging method has made it possible to detect cerebral $A \beta$ deposition in people without postmortem autopsy by using positron emission tomography (PET). Approximately $20-30 \%$ of CN elderly have AD-specific neuropathology in their brains as measured by carbon-11 labeled Pittsburgh compound-B $\left({ }^{11} \mathrm{C}-\mathrm{PiB}\right)$ PET [9]. However, these techniques have a few limitations. Employing amyloid PET is expensive, not commonly available and causes exposure to radiation. Otherwise, cerebrospinal fluid (CSF) analysis also can be used to gauge $A \beta 42$, however, an invasive lumbar puncture, labor intensiveness, and poor inter-laboratory reliability made it difficult to be implemented [10].

Neuropsychological assessment, sensitive and cost-efficient measurement for diagnosing AD, can be an evaluation tool for AD related pathology without invasive method. Despite several advantages in applying neuropsychological assessment for evaluating AD pathology, only a few studies denoted the results using the assessment as a predictive tool [11, 12]. A recent study demonstrated the predictability based on neuropsychological assessment and demographics using a brief machine learning (ML) approach [13]. In the study, the result using ML suggested that neuropsychological tests with demographics could be used as a preliminary screening for detecting $\mathrm{A} \beta$ before undergoing neuroimaging such as amyloid PET. Excepts for several studies, the majority of the findings that applied ML were studies using structural MRI images in the AD-related research fields [14, 15]. Additionally, there were few studies that focused on applying ML techniques to AD studies to predict $\mathrm{A} \beta$ status $[16,17]$.

The goal of the current study was to propose models that can predict abnormal level of cerebral $\mathrm{A} \beta$ based on neuropsychological test scores and demographics using several ML algorithms. For comparing predictability performance with neuropsychological test derived models, two brain measures were considered which were well known to be related with the cerebral amyloid deposition $[18,19]$ : cortical thickness and volume. Finally, feature selection approaches were selected to provide the explainable and practical implications for results.

\section{Materials and Methods}

\subsection{Ethics Statement}

In this study, we used participant data from ADNI, a multicenter project with approximately 50 medical centers and university sites across the United States and Canada [20]. ADNI was launched in 2003 as a public-private partnership led by principal investigator, Michael W. Weiner, MD. Its primary goal is to test whether biological markers such as serial MRI, PET, and clinical and neuropsychological assessment can be combined to measure the progression of patients with mild cognitive impairment (MCI) and early AD. Participants were between 55 and 90 years old, and were able to undergo all assessment procedures, and consent to participate in longitudinal followup. Written consent forms were obtained from all participants and the study was conducted after approval from Institutional Review Board at each participating institution.

\subsection{Participants}

$\mathrm{CN}$ participants were the control group in ADNI study and showed no significant clinical symptoms such as depression, MCI, or dementia. Participants with significant memory concern (SMC) score within the normal range for cognitive function but reported concerns about their memory. Participants with EMCI and LMCI reported their SMC either autonomously via an informant or through clinicians. However, daily living activities were preserved and other cognitive domains showed no significant impairment without signs of dementia. The degree of MCI (early or late) was determined with Wechsler Memory Scale Logical Memory II [21]. Participants with AD 
met National Institute of Neurological and Communicative Disorders and Stroke-Alzheimer's Disease and Related Disorders Association criteria for probable AD [22, 23]. A detailed description of the inclusion/exclusion criteria can be found at http://adni.loni.usc.edu/.

Data were downloaded from the ADNI database and included all subjects recruited in the ADNI-2 with complete baseline data available for cognitive assessment, APOE genotype processing and PET A $\beta$ quantitation. Our study sample included 724 subjects (170 control subjects, 95 with SMC, 324 with MCI (169 with EMCI and 155 with LMCI), and 135 with AD) who were recruited between 2011 and 2013, each of whom had a baseline APOE genotype, structural MRI, and florbetapir session.

\subsection{Amyloid PET data}

Baseline $A \beta$ deposition was visualized using florbetapir-PET. Amyloid PET results were downloaded from the latest available dataset "UCSF Berkeley - AV45 Analysis", Version: 2019-0827 excel in the ADNI database. Detailed methods for PET acquisition and analysis can be found from the works by Landau and colleges [24,25]. Florbetapir images consisted of $4 \times 5 \mathrm{~min}$ frames acquired 50-70 min after injection. Images were realigned, averaged, resliced to a common voxel size $(1.5 \mathrm{~mm})$, and smoothed to a common resolution of $8 \mathrm{~mm}$ in full width at half-maximum [26]. Structural T1-weighted images were acquired concurrently with the baseline florbetapir images. The images were used as structural templates to define the cortical regions of interest (ROIs), and the reference regions in native space for each subject were obtained with FreeSurfer software (version 5.3.0), which is documented and freely available on online (http://surfer.nmr.mgh. harvard.edu/). Baseline florbetapir scans for each participant were co-registered to baseline structural T1-weighted images. Images were subsequently used to extract weighted cortical retention indices, standardized uptake value (SUV) from gray matter within four cortical ROIs (frontal, anterior/posterior cingulate, lateral parietal, and lateral temporal) that were averaged to generate a mean cortical SUV as described online in greater detail(adni.bitbucket.org/docs/UCBERKELEYAV45/UCBERKELEY_AV45_Methods_12.03.15.pdf). Cortical SUV ratios (SUVR) were obtained by normalizing cortical SUV with the mean uptake in the whole cerebellum reference region. Participants were classified as cerebral A $\beta$ positive if the florbetapir SUV ratio was greater than 1.1.

\subsection{Structural MRI data}

Structural MRI scans were obtained at baseline visit according to a standardized protocol (http://adni.loni.usc.edu/about/centers-cores/mri-core/). Briefly, all participants in the current study were scanned using the 3T-MRI scanning protocol (GE, Philips or Siemens). Cortical reconstruction and volumetric segmentation were performed with the same FreeSurfer image analysis suite. Cortical thickness and volume data was downloaded from "UCSF - Cross-Sectional FreeSurfer (5.1), Version: 2019-11-08" excel in the ADNI database. Further details for MRI scans are described in more detail elsewhere [27].

\subsection{Neuropsychological assessment}

The following measurements were considered: Mini-Mental State Examination (MMSE) [28]; Alzheimer's Disease Assessment Scale (ADAS-Cog 13) [29]; Rey Auditory Verbal Learning Test (AVLT) [30]; Logical Memory (LM) [21]; Clock Drawing Task [31]; Trail Making Test [32]; Category Fluency [33]; Boston Naming Test (BNT) [31]; and American National Adults Reading Test (ANART) [34]. 


\subsection{Statistical analyses}

Demographics were compared between groups using t-test. $\chi 2$ tests were used to compare dichotomous variables. For predictability estimation, the aforementioned features, cortical thickness and volume, and cognitive performances were used as input data for supervised learning ML algorithm. Algorithms used in current study works as follows: logistic regression (LR), support vector machine (SVM), boosted decision tree (BDT), and artificial neural network (ANN).

Next, to explain multivariate profiles with respect to input features that were accurately distinguished from $\mathrm{A} \beta$ positivity for participants, the adaptive least absolute shrinkage and selection operator (LASSO) ML algorithm were applied to the dataset [35]. The adaptive LASSO, which is a penalized regression method [36], is a popular technique for simultaneous estimation and consistent variable selection [35]. The regression coefficients of unimportant variables shrank to 0 by implementing the adaptive LASSO. In that regard, the adaptive LASSO algorithm provided interpretable results related to abnormal levels of cerebral $\mathrm{A} \beta$ status.

In order to evaluate generalizability of the results from the ML algorithms, 10-fold crossvalidation was applied during the variable selection process. First, the data was randomly split into a training set $(66.7 \%$ of the data) and a test set $(33.4 \%$ of the data). All of the ML models were fitted using the training set and classifications were separately made on the test and training datasets. The optimal parameter, lambda, was determined across 1,000 iterations of 10-fold cross-validation to minimize the deviance of the model. Then, predictions were made on the test set based on the ML models trained in the training set.

The parameters used to compare predictability performances and validations of models were as follows: accuracy (ACC), precision (PRE), recall (REC), F1 score, the area under the curve (AUC) Analyses were performed using R, version 3.6.1 (http://www.r-project.org/).

\section{Results}

\subsection{Subject characteristics}

Demographic data for all participants are presented in Table 1. Of the 724 study participants, $339(46 \%)$ were $\mathrm{APOE} \varepsilon 4$ carriers, and $400(54 \%)$ were $\mathrm{A} \beta$ positive $(\mathrm{A} \beta+)$.

Table 1. Demographics of participants

\begin{tabular}{lllllcc}
\hline Characteristics & $\mathbf{C N}$ & $\begin{array}{l}\text { SMC } \\
\mathbf{( N = 1 7 0 )}\end{array}$ & $\begin{array}{l}\text { EMCI } \\
\mathbf{( N = 9 5 )}\end{array}$ & $\begin{array}{l}\text { LMCI } \\
\mathbf{( N = 1 6 9 )}\end{array}$ & $\begin{array}{c}\text { AD } \\
\mathbf{( N = 1 3 5 )}\end{array}$ & $\begin{array}{c}\text { Total } \\
\mathbf{( N = 7 2 4 )}\end{array}$ \\
\hline Age, years & $73.9(6.2)$ & $72.9(5.7)$ & $71.6(7.1)$ & $72.6(7.6)$ & $74.9(8.1)$ & $73.2(7.1)$ \\
No. of females (\%) & $91(51 \%)$ & $55(58 \%)$ & $71(42 \%)$ & $71(46 \%)$ & $56(41 \%)$ & $344(47 \%)$ \\
Education, year & $16.6(2.5)$ & $16.7(2.6)$ & $16.2(2.7)$ & $16.6(2.6)$ & $15.7(2.6)$ & $15.7(2.6)$ \\
No. of APOE $\varepsilon 4$ & $50(28 \%)$ & $29(31 \%)$ & $77(46 \%)$ & $90(58 \%)$ & $93(69 \%)$ & $339(46 \%)$ \\
carriers (\%) & & & & & & \\
A $\beta$ positivity (\%) & $53(29 \%)$ & $33(35 \%)$ & $84(50 \%)$ & $108(70 \%)$ & $122(90 \%)$ & $400(54 \%)$ \\
\hline
\end{tabular}

Abbreviations: APOE $\varepsilon 4$, apolipoprotein; $\mathrm{A} \beta$, amyloid-beta; $\mathrm{CN}$, clinically normal; $\mathrm{SMC}$, subjective memory concerns; EMCI, early mild cognitive impairment; $\mathrm{LMCI}$, late mild cognitive impairment; AD, Alzheimer's disease. Data are presented as mean (SD) unless otherwise indicated. aPOE $\varepsilon 4$ carriers are the percentage of individuals with at least one APOE $\varepsilon 4$ allele. 


\subsection{Feature combination}

In an attempt to compare the predictive performances of each model across combination of input features, we split the features into several subsets: 1) Whole dataset: Demographics (Demo) + neuropsychological assessment (NA) + cortical thickness (CT) + cortical column (CV);2) Demo + CV + NA; 3) Demo + CT + NA; 4) Demo + CV + CT; 5) Demo + NA.

Performance of the ML models

The results from each ML model are presented in Table 2. With respect to structural MRI information, applying SVM algorithm to each subset was likely to demonstrate slightly better performances than others. However, there were a few differences in performance metrics over subsets and applied algorithms. We can see that the results of 5th combination (Demo+NA) were not much different from the results using structural MRI information. Thus, 5th combination (Demo+NA) was selected as further consideration due to its cost-efficiency compared to MRI methods. Unlike previous results, applying the ANN algorithm showed better performance than the BDT algorithm (Table 3).

\subsection{Feature selection from Adaptive LASSO}

The predictability from demographic (age, gender, and APOE $\varepsilon 4$ status) and neuropsychological data was assessed for the performance to predict cerebral amyloid positivity. Figure 1 shows the multivariate profiles for cerebral A $\beta$ positivity, revealed by adaptive LASSO algorithm. First, A $\beta$ positivity was more prevalent in participants who were older, female, APOE $\varepsilon 4$ carriers, and showed poor cognitive performance in several measures in the ADNI-2 population (worse delayed recall and global cognition: ADAS-Cog 13). The receiver operating characteristic (ROC) demonstrated AUC of 0.824 for distinguishing $A \beta$ positivity using 6 of 20 features. 
Table 2. Results on models including MRI markers

\begin{tabular}{|c|c|c|c|c|c|}
\hline & $\mathrm{ACC}$ & RPE & REC & F1 & AUC \\
\hline \multicolumn{6}{|c|}{ WHOLE } \\
\hline LR & 0.75 & 0.78 & 0.76 & 0.77 & 0.84 \\
\hline SVM & 0.75 & 0.79 & 0.75 & 0.77 & 0.85 \\
\hline BDT & 0.76 & 0.81 & 0.72 & 0.77 & 0.85 \\
\hline ANN & 0.74 & 0.77 & 0.74 & 0.76 & 0.81 \\
\hline \multicolumn{6}{|c|}{$D e m o+C V+N A$} \\
\hline LR & 0.76 & 0.80 & 0.76 & 0.78 & 0.84 \\
\hline SVM & 0.76 & 0.80 & 0.74 & 0.77 & 0.85 \\
\hline BDT & 0.75 & 0.81 & 0.71 & 0.76 & 0.84 \\
\hline ANN & 0.73 & 0.77 & 0.73 & 0.75 & 0.81 \\
\hline \multicolumn{6}{|c|}{$\mathrm{Demo}+\mathrm{CT}+\mathrm{NA}$} \\
\hline LR & 0.75 & 0.79 & 0.75 & 0.77 & 0.84 \\
\hline SVM & 0.76 & 0.80 & 0.76 & 0.78 & 0.84 \\
\hline BDT & 0.77 & 0.83 & 0.74 & 0.78 & 0.82 \\
\hline ANN & 0.75 & 0.77 & 0.78 & 0.78 & 0.82 \\
\hline \multicolumn{6}{|c|}{$D e m o+C V+C T$} \\
\hline LR & 0.77 & 0.82 & 0.76 & 0.79 & 0.84 \\
\hline SVM & 0.74 & 0.80 & 0.72 & 0.76 & 0.84 \\
\hline BDT & 0.75 & 0.83 & 0.70 & 0.76 & 0.83 \\
\hline ANN & 0.71 & 0.85 & 0.58 & 0.69 & 0.81 \\
\hline
\end{tabular}

Abbreviations: WHOLE; Demp+NA+CT+CV; Demo: demographics; NA. neuropsychological assessment; CT. cortical thickness; CV. Cortical volume; ACC. accuracy; PRE. precision; REC. recall; AUC. area under curve; LR. Logical regression; SVM. support vector machine; BDT. boosted decision tree; ANN. artificial neural network. 
Table 3. Results on models excluding MRI markers

\begin{tabular}{lccccc}
\hline & ACC & RPE & REC & F1 & AUC \\
\hline Deom+NA & & & & & \\
LR & 0.75 & 0.79 & 0.74 & 0.77 & 0.83 \\
SVM & 0.75 & 0.80 & 0.73 & 0.76 & 0.83 \\
BDT & & & & & \\
ANN & 0.71 & 0.76 & 0.70 & 0.73 & 0.79 \\
& & & & & 0.83
\end{tabular}

Abbreviations: Demo: demographics; NA. neuropsychological assessment; CT. cortical thickness; CV. Cortical volume; ACC. accuracy; PRE. precision; REC. recall; AUC. area under curve; LR. Logical regression; SVM. support vector machine; BDT. boosted decision tree; ANN. artificial neural network.

Figure 1. Multivariate patterns of demographic information and neuropsychological markers predicting cerebral a $\beta$ burden: ADAS13, Alzheimer's disease assessment scale; APOE, ApoE $\varepsilon 4$ positivity; AVLT _Del, Rey auditory verbal learning test delayed recall; LM_del, logical memory delayed recall.

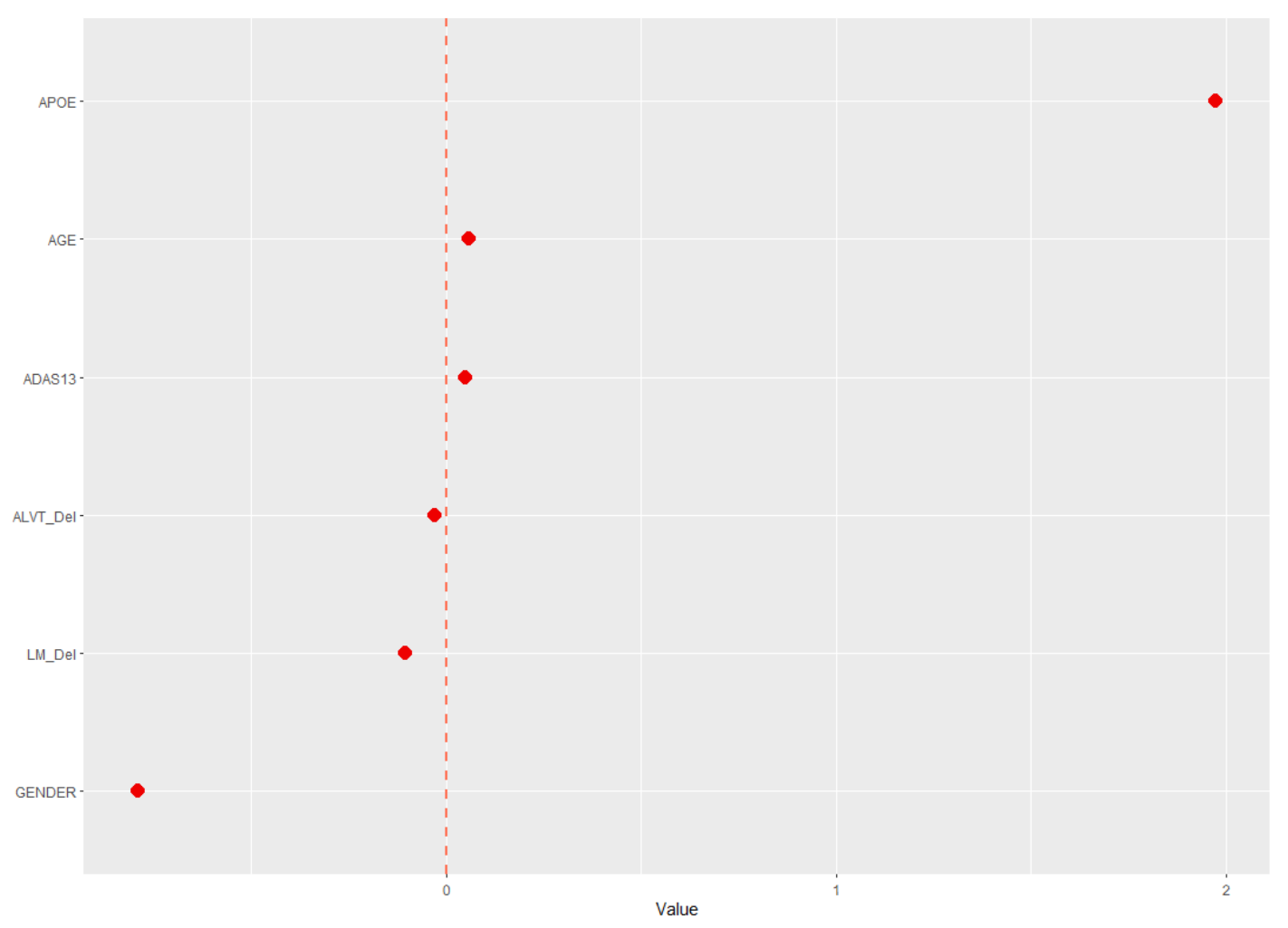


Figure 2. Receiver operating characteristic (ROC) curve based on the adaptive LASSO result.

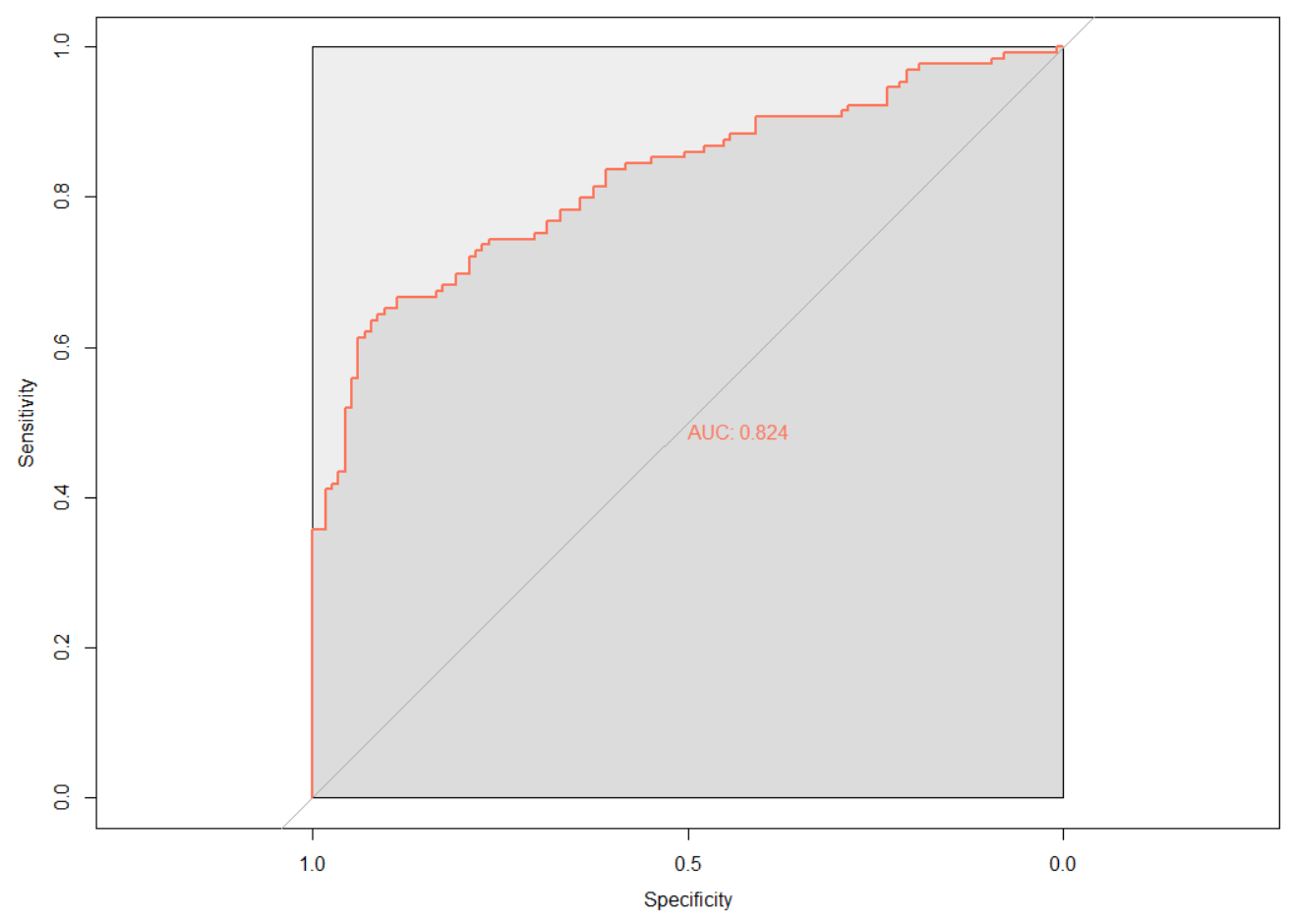

\section{Discussion}

Present study proposed a ML approach using various classifiers based on demographics, cortical thickness and volume, and neuropsychological performance data to predict cerebral A $\beta$ deposition in the ADNI-2 cohort. The subset using demographics and neuropsychological assessment data demonstrated better cost-efficiency compared to other models including structural MRI data. Therefore, the cost-efficient model was chosen for further analysis, and the adaptive LASSO algorithm was adopted in feature selection process which can be the most predictive measure for cerebral A $\beta$ positivity. Finally, multivariate profiles of cognitive performances and demographics were used to support explainability of the model.

Although some studies have reported high predictive accuracy for A $\beta$ positivity based on ML methods, these studies were based on blood biomarkers or combined neuropsychological tests [16, 17]. Only one recent study showed the predictability for $A \beta$ status using neuropsychological assessment based on ML [13]. To our knowledge, this is one of the first studies that evaluated relative performances using ML models predicting cerebral amyloid status based on several ML algorithms in the AD research.

Current findings demonstrate that including structural MRI markers can predict A $\beta$ positivity with $85 \%$ predictability (Demo+CV+NA model using SVM, Table 2) in the ADNI-2 participants. The cost-efficient model (Demo+NA), however, shows similar results (Table 3), leading to the same result using adaptive Lasso model (AUC $=0.85$ ). Given these results, specific neuropsychological measures, even without neuroimaging markers, have practical implications for prediction of neuropathological biomarkers in AD without invasive methods (i.e. PET or CSF method).

In ADNI-2 cohort, age, gender, and APOE $\varepsilon 4$ status are significant variables to predict $\mathrm{A} \beta$ positivity. However, education is not a significant indicator. These results are consistent with previous studies [37-39]. It is remarkable that certain cognitive measures show higher coefficient in predicting $A \beta$ positivity compared to that of age, which is consistent with previous studies $[13,40]$. 
As mentioned earlier, most of the studies using ML approaches have focused on diagnosis and progression of the disease based on limited biomarkers such as volumetric and/or surface-based brain measures, or blood proteins in AD research [14, 15, 41]. Based on the first study, our current work proposed the implication in ML-based models that can predict $A \beta$ positivity, suggesting potential application of $\mathrm{A} \beta$ assessment through non-invasive MRI markers. Since the NIA-AA research framework has been updated, neuroimaging markers derived from structural MRI scans have been considered as non-specific information about neuronal injury and neurodegenerative changes in the AD course [42]. However, our results represented that MRI markers may still have beneficial information for AD specific pathology with combination of other layers of information such as demographics and neuropsychological assessments. Given the fact that employing amyloid PET scans has relatively limited accessibility than that of MRI scans, predicting cerebral A $\beta$ status using MRI scans may provide potential implications in the health care system. First, progress of AD pathology can be predicted during assessment of other brain diseases with automated ML algorithm to on-line structural MRI analysis. Second, it could be an alternative modality for assessing AD specific pathology in case of the use of amyloid PET scans is limited.

More importantly, our findings demonstrate that the results based on neuropsychological data with several demographics is nearly accurate as the MRI markers in predicting cerebral A $\beta$ status. This suggests the following benefits. Given that in most AD-related clinical trials, delayed recall and global cognition assessment are administrated as screening tools for participants' inclusion, a combination of these tests can be a preliminary tool in predicting $A \beta$ status. There are financial advantages on detecting cerebral A $\beta$ without Amyloid PET scans, considering the high cost of amyloid pet scan (\$5,000 per person in the US; [43]). Moreover, the explainable profile from the adaptive lasso results can provide clinicians with reliable information about AD-specific pathology.

The current study has several limitations. First, ADNI population is not representative of the general population because the participants were deliberately selected based on robust inclusion criteria. Moreover, most participants in ADNI-2 cohort were recruited only from North America. Second, the results were only estimated in baseline visit, hence, the cross-sectional results are limited for causal assumptions of the input features and the outcome variables. In this regard, 10fold-cross validation for out-of-sample generalization may support generalization of the results. Third, the use of limited structural MRI markers may not have enriched the results, suggesting that consideration of other MRI markers such as resting state fMRI, hippocampal volume, or T2weighted image markers are needed to be considered for further analysis. Finally, although the results may cause an over-fitting problem regarding high dimensionality in structural MRI data, the process of dimension reduction is not implemented. Dimension reduction is not performed in order to prevent loss of information because it is pre-processed by Freesurfer suite rather than 2D image data. Undoubtedly, since the feature selection procedure for the final model serves as a dimensionality reduction, it is assumed that the cost-efficient model could have avoided overfitting problems. Further research should be validated against various cohort studies.5.

\section{Conclusions}

In conclusion, our findings demonstrate that MRI markers using an ML algorithm might predict abnormal level of $\mathrm{A} \beta$ status in ADNI-2 cohort without invasive methods. Results also provide the same predictability using neuropsychological model based on the adaptive LASSO methods, which is cost-efficient than conventional neuroimaging methods. Application of these findings may give us opportunity to develop precise intervention for the patients who suffer from dementia. The costefficient, non-invasive, and easily accessible aspects of the method can contribute to thorough recruitment for $\mathrm{AD}$ clinical trials and serve as an alternative method to patients who have difficulties in undergoing PET scans. 
Author Contributions: All authors listed, have made substantial, direct and intellectual contribution to the work, and approved it for publication.

Funding: This research was supported by the Ministry of Science and ICT, Korea, under the Information Technology Research Center support program (No. IITP- 2020-2017-0-01630) supervised by the Institute for Information \& communications Technology Promotion (IITP).

Conflicts of Interest: The authors declare no conflict of interest.

\section{References}

1. Organization, W.H., Dementia: a public health priority. 2012: World Health Organization.

2. Association, A.s., 2016 Alzheimer's disease facts and figures. Alzheimer's \& Dementia, 2016. 12(4): p. 459509.

3. Plassman, B.L., K.M. Langa, G.G. Fisher, S.G. Heeringa, D.R. Weir, M.B. Ofstedal, J.R. Burke, M.D. Hurd, G.G. Potter, and W.L. Rodgers, Prevalence of dementia in the United States: the aging, demographics, and memory study. Neuroepidemiology, 2007. 29(1-2): p. 125-132.

4. Frisoni, G.B., N.C. Fox, C.R. Jack Jr, P. Scheltens, and P.M. Thompson, The clinical use of structural MRI in Alzheimer disease. Nature Reviews Neurology, 2010. 6(2): p. 67.

5. Jack Jr, C.R., D.S. Knopman, W.J. Jagust, L.M. Shaw, P.S. Aisen, M.W. Weiner, R.C. Petersen, and J.Q. Trojanowski, Hypothetical model of dynamic biomarkers of the Alzheimer's pathological cascade. The Lancet Neurology, 2010. 9(1): p. 119-128.

6. Braak, H. and E. Braak, Neuropathological stageing of Alzheimer-related changes. Acta neuropathologica, 1991. 82(4): p. 239-259.

7. Silbert, L.C., J. Quinn, M. Moore, E. Corbridge, M. Ball, G. Murdoch, G. Sexton, and J. Kaye, Changes in premorbid brain volume predict Alzheimer's disease pathology. Neurology, 2003. 61(4): p. 487-492.

8. Jack Jr, C.R., D.S. Knopman, W.J. Jagust, R.C. Petersen, M.W. Weiner, P.S. Aisen, L.M. Shaw, P. Vemuri, H.J. Wiste, and S.D. Weigand, Tracking pathophysiological processes in Alzheimer's disease: an updated hypothetical model of dynamic biomarkers. The Lancet Neurology, 2013. 12(2): p. 207-216.

9. Aizenstein, H.J., R.D. Nebes, J.A. Saxton, J.C. Price, C.A. Mathis, N.D. Tsopelas, S.K. Ziolko, J.A. James, B.E. Snitz, and P.R. Houck, Frequent amyloid deposition without significant cognitive impairment among the elderly. Archives of neurology, 2008. 65(11): p. 1509-1517.

10. Mattsson, N. and H. Zetterberg, Alzheimer's disease and CSF biomarkers: key challenges for broad clinical applications. Biomarkers in medicine, 2009. 3(6): p. 735-737.

11. Curiel, R.E., D. Loewenstein, E. Crocco, M. Greig-Custo, R. Rodriquez, S. Czaja, M. Rosado, W.W. Barker, and R. Duara, A novel measure of cognitive change in preclinical Alzheimer's disease and its physiological correlates in normal and MCI elderly. Alzheimer's \& Dementia: The Journal of the Alzheimer's Association, 2015. 11(7): p. P822.

12. Schindler, S.E., M.S. Jasielec, H. Weng, J.J. Hassenstab, E. Grober, L.M. McCue, J.C. Morris, D.M. Holtzman, C. Xiong, and A.M. Fagan, Neuropsychological measures that detect early impairment and decline in preclinical Alzheimer disease. Neurobiology of aging, 2017. 56: p. 25-32.

13. Ko, H., J.-J. Ihm, H.-G. Kim, and A.s.D.N. Initiative, Cognitive Profiling Related to Cerebral Amyloid Beta Burden Using Machine Learning Approaches. Frontiers in aging neuroscience, 2019. 11.

14. Moradi, E., A. Pepe, C. Gaser, H. Huttunen, J. Tohka, and A.s.D.N. Initiative, Machine learning framework for early MRI-based Alzheimer's conversion prediction in MCI subjects. Neuroimage, 2015. 104: p. 398-412. 
15. Salvatore, C., A. Cerasa, P. Battista, M.C. Gilardi, A. Quattrone, and I. Castiglioni, Magnetic resonance imaging biomarkers for the early diagnosis of Alzheimer's disease: a machine learning approach. Frontiers in neuroscience, 2015. 9: p. 307.

16. Burnham, S., N. Faux, W. Wilson, S. Laws, D. Ames, J. Bedo, A. Bush, J. Doecke, K. Ellis, and R. Head, A blood-based predictor for neocortical A $\beta$ burden in Alzheimer's disease: results from the AIBL study. Molecular psychiatry, 2014. 19(4): p. 519.

17. Haghighi, M., A. Smith, D. Morgan, B. Small, and S. Huang, Identifying cost-effective predictive rules of amyloid- $\beta$ level by integrating neuropsychological tests and plasma-based markers. Journal of Alzheimer's Disease, 2015. 43(4): p. 1261-1270.

18. Becker, J.A., T. Hedden, J. Carmasin, J. Maye, D.M. Rentz, D. Putcha, B. Fischl, D.N. Greve, G.A. Marshall, and S. Salloway, Amyloid- $\beta$ associated cortical thinning in clinically normal elderly. Annals of neurology, 2011. 69(6): p. 1032-1042.

19. Storandt, M., M.A. Mintun, D. Head, and J.C. Morris, Cognitive decline and brain volume loss as signatures of cerebral amyloid- $\beta$ peptide deposition identified with Pittsburgh compound B: cognitive decline associated with A $\beta$ deposition. Archives of neurology, 2009. 66(12): p. 1476-1481.

20. Petersen, R.C., P. Aisen, L.A. Beckett, M. Donohue, A. Gamst, D.J. Harvey, C. Jack, W. Jagust, L. Shaw, and A. Toga, Alzheimer's disease neuroimaging initiative (ADNI): clinical characterization. Neurology, 2010. 74(3): p. 201-209.

21. Wechsler, D., WMS-R: Wechsler memory scale-revised: manual. 1984: Psychological Corporation.

22. Dubois, B., H.H. Feldman, C. Jacova, S.T. DeKosky, P. Barberger-Gateau, J. Cummings, A. Delacourte, D. Galasko, S. Gauthier, and G. Jicha, Research criteria for the diagnosis of Alzheimer's disease: revising the NINCDS-ADRDA criteria. The Lancet Neurology, 2007. 6(8): p. 734-746.

23. McKhann, G., D. Drachman, M. Folstein, R. Katzman, D. Price, and E.M. Stadlan, Clinical diagnosis of Alzheimer's disease Report of the NINCDS-ADRDA Work Group* under the auspices of Department of Health and Human Services Task Force on Alzheimer's Disease. Neurology, 1984. 34(7): p. 939-939.

24. Landau, S.M., C. Breault, A.D. Joshi, M. Pontecorvo, C.A. Mathis, W.J. Jagust, and M.A. Mintun, Amyloid- $\beta$ imaging with Pittsburgh compound B and florbetapir: comparing radiotracers and quantification methods. Journal of nuclear medicine: official publication, Society of Nuclear Medicine, 2013. 54(1): p. 70 .

25. Landau, S.M., M.A. Mintun, A.D. Joshi, R.A. Koeppe, R.C. Petersen, P.S. Aisen, M.W. Weiner, W.J. Jagust, and A.s.D.N. Initiative, Amyloid deposition, hypometabolism, and longitudinal cognitive decline. Annals of neurology, 2012. 72(4): p. 578-586.

26. Joshi, A., R.A. Koeppe, and J.A. Fessler, Reducing between scanner differences in multi-center PET studies. Neuroimage, 2009. 46(1): p. 154-159.

27. Hartig, M., D. Truran-Sacrey, S. Raptentsetsang, A. Simonson, A. Mezher, N. Schuff, and M. Weiner, UCSF freesurfer methods. ADNI: Alzheimers Disease Neuroimaging Initiative, San Francisco, 2014.

28. Folstein, M.F., S.E. Folstein, and P.R. McHugh, "Mini-mental state": a practical method for grading the cognitive state of patients for the clinician. Journal of psychiatric research, 1975. 12(3): p. 189-198.

29. Mohs, R.C., D. Knopman, R.C. Petersen, S.H. Ferris, C. Ernesto, M. Grundman, M. Sano, L. Bieliauskas, D. Geldmacher, and C. Clark, Development of cognitive instruments for use in clinical trials of antidementia drugs: additions to the Alzheimer's Disease Assessment Scale that broaden its scope. Alzheimer disease and associated disorders, 1997.

30. Rey, A., The clinical examination in psychology. Paris: Presses Universitaires de France, 1964.

31. Kaplan, E., The assessment of aphasia and related disorders. Vol. 2. 1983: Lippincott Williams \& Wilkins. 
32. Reitan, R.M. and D. Wolfson, The Halstead-Reitan Neuropsychological Test Battery. 1986.

33. Morris, J., A. Heyman, R. Mohs, J. Hughes, G. Van Belle, G. Fillenbaum, E. Mellits, and C. Clark, The consortium to establish a registry for Alzheimer's disease (CERAD): I. Clinical and neuropsychological assessment of Alzheimer's disease. Neurology, 1989.

34. Grober, E., M. Sliwinsk, and S.R. Korey, Development and validation of a model for estimating premorbid verbal intelligence in the elderly. Journal of clinical and experimental neuropsychology, 1991. 13(6): p. 933949.

35. Zou, H., The adaptive lasso and its oracle properties. Journal of the American statistical association, 2006. 101(476): p. 1418-1429.

36. Tikhonov, A.N. On the stability of inverse problems. in Dokl. Akad. Nauk SSSR. 1943.

37. Jansen, W.J., R. Ossenkoppele, D.L. Knol, B.M. Tijms, P. Scheltens, F.R. Verhey, P.J. Visser, P. Aalten, D. Aarsland, and D. Alcolea, Prevalence of cerebral amyloid pathology in persons without dementia: a metaanalysis. Jama, 2015. 313(19): p. 1924-1938.

38. Lee, J.H., M.S. Byun, D. Yi, Y.M. Choe, H.J. Choi, H. Baek, B.K. Sohn, J.-Y. Lee, H.J. Kim, and J.W. Kim, Sex-specific association of sex hormones and gonadotropins, with brain amyloid and hippocampal neurodegeneration. Neurobiology of aging, 2017. 58: p. 34-40.

39. Morris, J.C., C.M. Roe, C. Xiong, A.M. Fagan, A.M. Goate, D.M. Holtzman, and M.A. Mintun, APOE predicts amyloid-beta but not tau Alzheimer pathology in cognitively normal aging. Annals of neurology, 2010. 67(1): p. 122-131.

40. Mortamais, M., J.A. Ash, J. Harrison, J. Kaye, J. Kramer, C. Randolph, C. Pose, B. Albala, M. Ropacki, and C.W. Ritchie, Detecting cognitive changes in preclinical Alzheimer's disease: A review of its feasibility. Alzheimer's \& Dementia, 2017. 13(4): p. 468-492.

41. Casanova, R., S. Varma, B. Simpson, M. Kim, Y. An, S. Saldana, C. Riveros, P. Moscato, M. Griswold, and D. Sonntag, Blood metabolite markers of preclinical Alzheimer's disease in two longitudinally followed cohorts of older individuals. Alzheimer's \& Dementia, 2016. 12(7): p. 815-822.

42. Jack Jr, C.R., D.A. Bennett, K. Blennow, M.C. Carrillo, B. Dunn, S.B. Haeberlein, D.M. Holtzman, W. Jagust, F. Jessen, and J. Karlawish, NIA-AA Research Framework: Toward a biological definition of Alzheimer's disease. Alzheimer's \& Dementia, 2018. 14(4): p. 535-562.

43. O'Bryant, S.E., M.M. Mielke, R.A. Rissman, S. Lista, H. Vanderstichele, H. Zetterberg, P. Lewczuk, H. Posner, J. Hall, and L. Johnson, Blood-based biomarkers in Alzheimer disease: current state of the science and a novel collaborative paradigm for advancing from discovery to clinic. Alzheimer's \& Dementia, 2017. 13(1): p. $45-58$. 